\title{
Prognostic models for stage I-III esophageal cancer: a comparison between existing calculators
}

\author{
Riccardo Lemini ${ }^{1}$, Tamara Díaz Vico ${ }^{1}$, Denslow A. Trumbull ${ }^{2}$, Kristopher Attwood ${ }^{3}$, Aaron C. Spaulding ${ }^{4}$, \\ Enrique F. Elli ${ }^{1}$, Dorin T. Colibaseanu ${ }^{1}$, Moshim Kukar ${ }^{5}$, Emmanuel Gabriel ${ }^{1}$ \\ ${ }^{1}$ Department of Surgery, Mayo Clinic, Jacksonville, FL, USA; ${ }^{2}$ University of Florida, College of Medicine, Gainesville, FL, USA; ${ }^{3}$ Department of \\ Biostatistics, Roswell Park Comprehensive Cancer Center, Buffalo, NY, USA; ${ }^{4}$ Department of Health Sciences Research, Mayo Clinic, Jacksonville, \\ FL, USA; ${ }^{5}$ Department of Surgical Oncology, Roswell Park Comprehensive Cancer Center, Buffalo, NY, USA \\ Contributions: (I) Conception and design: E Gabriel, M Kukar, DT Colibaseanu, EF Elli, AC Spaulding; (II) Administrative support: E Gabriel, M \\ Kukar, DT Colibaseanu, EF Elli, AC Spaulding; (III) Provision of study materials or patients: R Lemini, T Díaz Vico, DA Trumbull; (IV) Collection \\ and assembly of data: R Lemini, T Díaz Vico, DA Trumbull; (V) Data analysis and interpretation: K Attwood, R Lemini, T Díaz Vico, DA Trumbull; \\ (VI) Manuscript writing: All authors; (VII) Final approval of manuscript: All authors. \\ Correspondence to: Emmanuel Gabriel, MD, PhD, FACS, FSSO. Division of Surgical Oncology, Department of Surgery, Mayo Clinic, 4500 San Pablo \\ Rd., Jacksonville, FL 32224, USA. Email: gabriel.emmanuel@mayo.edu.
}

\begin{abstract}
Background: Determining the best approach for esophageal cancer and predicting accurate prognosis are critical. Multiple studies evaluated characteristics associated with overall survival, and several prediction models have been developed. This study aimed to evaluate existing models and perform external validation of selected models.
\end{abstract}

Methods: A retrospective investigation of a multi-site institutional enterprise for patients with a diagnosis of esophageal cancer between 2013-2014 was performed. Selected survival prediction models included the Roswell Park Comprehensive Cancer Center (RPCCC) calculator, Oregon Health \& Science University (OHSU) calculator, and two nomograms published by Shapiro et al. and Sun et al. One-year overall survival, level of agreement, and performance for each model were evaluated.

Results: A total of 104 patients were included and used to assess the prediction models. One-year overall survival was 0.76. Different calculators tended to rank patients similarly; however, they did not agree on predicted overall survival. The least disparity in correlation was observed between OHSU and Shapiro calculators. Shapiro's model achieved the highest performance [area under the curve (AUC) $=0.63$ ] .

Conclusions: Selected models showed fair results in estimating individual overall survival, although none achieved a high performance. While these tools may support the decision-making process for esophageal cancer patients, their implementation in clinical practice requires improved refinement to optimize their clinical utility.

Keywords: Esophageal cancer; overall survival (OS); calculator; nomogram; neoadjuvant chemoradiation

Submitted Aug 17, 2020. Accepted for publication Feb 15, 2021.

doi: 10.21037/jgo-20-337

View this article at: https://dx.doi.org/10.21037/jgo-20-337

\section{Introduction}

Esophageal cancer (EC) is the sixth leading cause of cancer-related death and the eighth most common cancer worldwide (1). It is one of the most lethal tumors and is considered to have significantly poorer prognosis than other gastrointestinal neoplasms. In 2018, 17,290 new EC cases and 15,850 EC-related deaths were estimated in the United States (2).

Although the incidence of esophageal squamous cell carcinoma remains stable, the incidence of esophageal adenocarcinoma (EA) has increased dramatically in the 
developed world in the last half century, positioning itself as the most dominant histological subtype of EC (3). There seem to be clear environmental influences, and predisposing factors (such as obesity, gastroesophageal reflux and Barrett's esophagus) also play an essential role in the natural history of EA (4-6).

Although the pathogenesis of EC is not fully defined, the underlying molecular changes are being investigated (7). Accordingly, there have been advances in the management of EC that have led to clinically relevant improvements in patient outcomes. Modern neoadjuvant chemoradiotherapy (CRT) followed by esophagectomy has surfaced as a promising approach, for stage II and III patients, to downstage and achieve pathological complete response (PCR), while improving overall survival (OS) (8-11). The most important randomized trial was performed by the Chemoradiation for Oesophageal Cancer Followed by Surgery Study (CROSS) group, which reported improved outcomes in terms of complete resection (R0) and median OS for patients with esophageal or esophagogastricjunction cancer with stages cT1N1 and cT2-3N0-1 after preoperative weekly administration of carboplatin, paclitaxel and concurrent radiotherapy compared with the surgery group alone (8). Currently, the CROSS trial represents the benchmark for the standard of care in patients with EC.

However, determining the most accurate therapeutic strategy for each patient remains challenging, and predicting survival represents a critical aspect for patients diagnosed with cancer. The presence of lymph node metastasis has been shown to be a significant prognosis factor for survival in EC patients $(12,13)$. In addition to the stage of the disease, the response to therapy has also been reported to be strongly associated with survival rate (14-16). Nonetheless, these sole characteristics are not sufficient to estimate accurate prognosis for individual patients as multiple other patient-, tumor- and treatment-related factors are important predictors for OS $(17,18)$.

To address this gap, several calculators and nomograms have been developed for patients diagnosed with EC (19-25). In order to increase accuracy, some of them have been designed for specific disease (metastatic or non-metastatic) or population (surgical vs non-surgical patients) settings. The following models, for instance, have been developed to assess individual OS for patients diagnosed with nonmetastatic EC who undergo surgery after neoadjuvant CRT (nCRT): (I) Roswell Park Comprehensive Cancer Center (RPCCC) calculator (20); (II) Oregon Health \& Science University (OHSU) calculator (19), and two nomograms published by (III) Shapiro et al. (22) and (IV) Sun et al. (23), respectively. Although the target population is similar, these models differ in the number and type of variables included to predict survival. Therefore, concordance among them can be often variable. The aims of this current study are to evaluate these chosen calculators and demonstrate their accuracy and performance in predicting OS for individual patients with stage Ib-III EC. We present the following article in accordance with the STROBE reporting checklist (available at https://dx.doi.org/10.21037/jgo-20-337).

\section{Methods}

This retrospective analysis of a multi-site institutional experience was performed for patients with a diagnosis of clinical stage Ib-III EC. We selected calculators or nomograms that included EA and those that had surgery with or without neoadjuvant chemotherapy or radiation therapy as part of the multidisciplinary management of EA. Therefore, surgery and receipt of neoadjuvant therapy had to be included in the calculator or nomogram variables. We also excluded tools derived only from the treatment of squamous cell cancer as well as tools that did not explicitly include neoadjuvant chemotherapy, in order to provide more homogeneity and generalizability within the comparative analysis. The tools that met these criteria included the RPCCC and OHSU calculators and the nomograms developed by Shapiro et al. and Sun et al.

The selection of patients was limited to diagnoses made from January 2013 to December 2014 because patients from the National Cancer Data Base (NCDB) between 2006 and 2012 are included in the RPCCC calculator. Thus, we required an external cohort of patients outside the years used in the above-mentioned calculator due to Mayo Clinic's participation in the NCDB in order to perform an appropriate external validation. This aspect did not affect the other calculators since they were developed using either patients prior to 2011 or from different databases. Specifically, the OHSU calculator and the nomogram published by Sun were based on the Surveillance Epidemiology and End Results (SEER) and were comprised of patients from 1995 to 2007 and 2006 to 2012 , respectively $(19,23)$. The nomogram published by Shapiro was instead based on patients recruited in CROSS-I (2001-2004) and CROSS-II (2004-2009) trials, and patients treated at the Erasmus MC in Rotterdam or at the Academic Medical Centre in Amsterdam (post-CROSS, 2009-2013). 


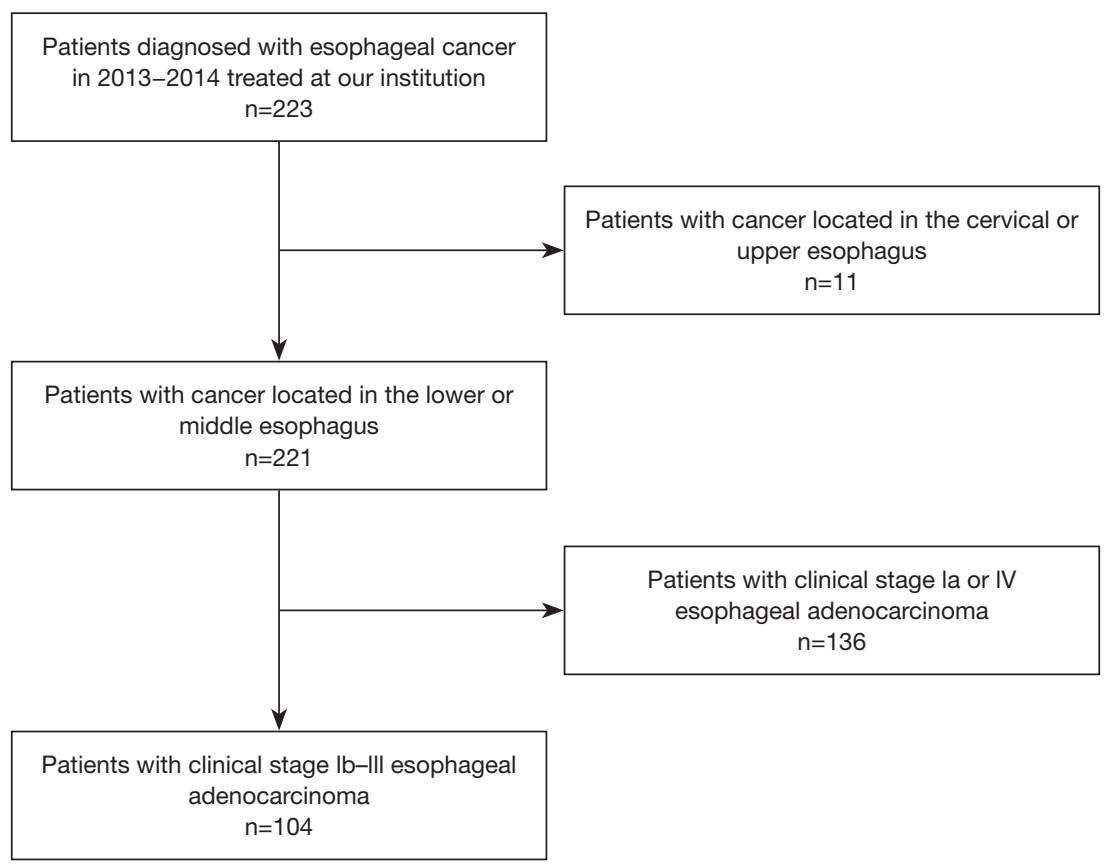

Figure 1 Inclusion and exclusion criteria used for patient selection for the comparison of the calculators.

Patients with clinical stage Ib-III adenocarcinoma of the middle and lower third of the esophagus who underwent surgery were included in the analysis. Patients with cancer located in the cervical and/or upper third of esophagus, carcinoma in situ, and more than one recorded malignancy were excluded from the study. Figure 1 outlines our study's inclusion and exclusion criteria.

The following variables were collected: age at diagnosis, sex, marital status, Charlson-Deyo comorbidity score, histology, clinical and pathological $\mathrm{T}$ and $\mathrm{N}$ stages, tumor grade, number of harvested lymph nodes, chemotherapy, radiotherapy, and surgery status. Table 1 summarizes the selected OS calculators' specific variables.

\section{Statistical analysis}

The subject characteristics were summarized using the mean, median, and standard deviation for continuous variables; and using frequencies and relative frequencies for categorical variables. Standard Kaplan-Meier methods were used to summarized OS; where estimates of median and $1-/ 3$-year survival were achieved with $95 \%$ confidence intervals.

Each calculator's survival scores were summated using the mean, median, and standard deviation. A scatter plot matrix was used to visually examine the association between scores from different calculators, while the intra-class correlation coefficient (ICC) assessed their agreement. Each model's performance was assessed separately using the area under the 1-year receiver operating characteristic (ROC) curve (AUC) and calibration plot. No formal comparison was made across models. All analyses were conducted at a significance level of 0.05 in SAS v9.4 (Cary, NC, USA).

\section{Ethical statement}

The study was conducted in accordance with the Declaration of Helsinki (as revised in 2013). This study was deemed exempt from the Mayo Clinic Florida Institutional Review Board (IRB), and informed consent was waived by our IRB as this retrospective study was deemed minimal risk to patients.

\section{Results}

Following the application of the selection criteria, a total of 104 patients were included. The overall patient, tumor, and treatment characteristics are listed in Table 2. The KaplanMeier curve in Figure 2 shows OS for all comers and based on clinical stage. OS data were available for all 104 patients, 
Table 1 Variables needed to calculate survival rates for each calculator

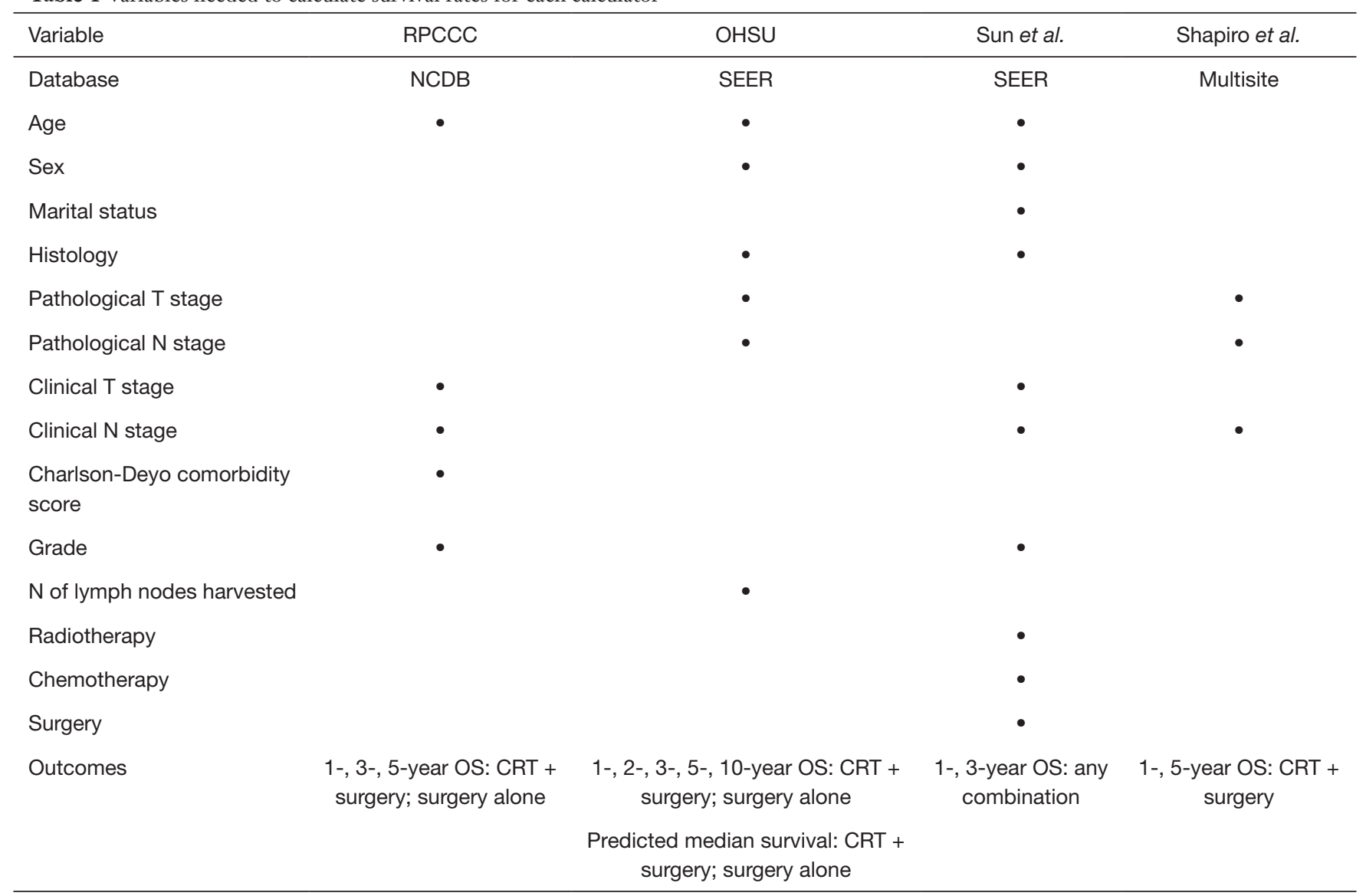

RPCCC, Roswell Park Comprehensive Cancer Center; OHSU, Oregon Health State University; NCDB, National Cancer Data Base; SEER, Surveillance Epidemiology and End Results; OS, overall survival; CRT, chemoradiation therapy.

of which 56 experienced an event (morality). The median survival time was 37.5 months [95\% confidence interval (CI): 26.3-52.2], and the 1-year OS was 0.76 (95\% CI: $0.67-0.84)$. The median follow-up was 48.8 months.

Figure 3 shows the scatter plot matrix used to evaluate the agreement for each calculator. When examining how the survival scores performed individually, the scatter plot matrix indicates that patients were likely to be ranked similarly among the different calculators. That is, if a patient received the highest survival score using the Roswell Park calculator, then that patient tended to get one of the highest scores on the other three calculators, as indicated by the Spearman correlation coefficients. Interestingly, the calculators did not agree on predicted survival estimates, as indicated by the low ICC. The OHSU and Shapiro risk calculators appeared to have a reasonable level of agreement, but the other calculators had limited agreement. The highest level of agreement was observed between
OHSU and Shapiro calculators (ICC 0.719), while the lowest occurred between OHSU and RPCCC calculators (ICC -0.055), which was due to the survival scores of RPCCC being the most optimistic.

The analysis of the performance of the calculators is reported in Figure 4. All the calculators performed similarly (AUC: $\min 0.5280-\max 0.6313$ ), with Shapiro calculator achieving the highest performance (AUC $=0.6313$ ). It is important to note that no formal statistical comparison of these 4 calculators was performed.

\section{Discussion}

The development of different models estimating OS has increased in the recent years, and their performance can provide useful information for the decision-making process and a more tailored approach to patient care $(19,20,22-30)$. In this study, an external validation and comparison between selected 
Table 2 Baseline characteristics used to compare survival calculators

\begin{tabular}{|c|c|c|}
\hline Characteristics & Subcategory & Overall, n (\%) \\
\hline Overall & $\mathrm{N}$ & $104(100.0)$ \\
\hline Age (years) & Mean/Std. & $63.3 / 9.7$ \\
\hline \multirow[t]{2}{*}{ Sex } & Male & $93(89.4)$ \\
\hline & Female & $11(10.6)$ \\
\hline \multirow[t]{2}{*}{ Marital status } & Married & $87(83.7)$ \\
\hline & Single & $17(16.3)$ \\
\hline \multirow[t]{3}{*}{ Charlson-Deyo score } & 0 & $18(17.3)$ \\
\hline & 1 & $35(33.7)$ \\
\hline & 2 & $51(49.0)$ \\
\hline \multirow[t]{4}{*}{ Clinical T } & 1 & $14(13.5)$ \\
\hline & 2 & $23(22.1)$ \\
\hline & 3 & $64(61.5)$ \\
\hline & 4 & $3(2.9)$ \\
\hline \multirow[t]{4}{*}{ Clinical N } & 0 & $37(35.6)$ \\
\hline & 1 & $50(48.1)$ \\
\hline & 2 & $14(13.5)$ \\
\hline & 3 & $3(2.9)$ \\
\hline \multirow[t]{5}{*}{ Pathological T } & 0 & $20(19.2)$ \\
\hline & 1 & $28(26.9)$ \\
\hline & 2 & $19(18.3)$ \\
\hline & 3 & $36(34.6)$ \\
\hline & 4 & $1(1.0)$ \\
\hline \multirow[t]{4}{*}{ Pathological N } & 0 & $61(58.7)$ \\
\hline & 1 & $22(21.2)$ \\
\hline & 2 & $16(15.4)$ \\
\hline & 3 & $5(4.8)$ \\
\hline \multirow[t]{4}{*}{ Grade } & 1 & $5(4.9)$ \\
\hline & 2 & $37(35.9)$ \\
\hline & 3 & $60(58.3)$ \\
\hline & 4 & $1(1.0)$ \\
\hline \multirow[t]{2}{*}{ Nodes examined } & Mean/Std. & $22.7 / 12.6$ \\
\hline & Median/Min./Max. & $20.0 / 3.0 / 84.0$ \\
\hline \multirow[t]{2}{*}{ Nodes positive } & Mean/Std. & $1.6 / 3.0$ \\
\hline & Median/Min./Max. & $0.0 / 0.0 / 17.0$ \\
\hline \multirow[t]{2}{*}{ Neoadjuvant CRT } & No & $5(4.8)$ \\
\hline & Yes & $99(95.2)$ \\
\hline
\end{tabular}

CRT, chemoradiation therapy. existing calculators was performed with the intention of achieving an evaluation of their accuracy. Moreover, the comparison among calculators describes which oncologic specific factors were most reliable for creating an accurate model.

The Shapiro calculator achieved the best performance when examining the performance of the calculators in estimating 1 -year survival rates (AUC $=0.63$ ). Herein, Shapiro et al. (22) used the pretreatment $\mathrm{cN}$ category and post-treatment ypN and yp $\mathrm{T}$ categories to develop their nomogram. In accordance to previous studies, the number of lymph nodes with metastasis has shown to be strongly associated with survival in patients with EA who receive preoperative chemoradiation $(31,32)$. The OHSU risk calculator achieved a reasonable performance, being the second most accurate one (AUC $=0.56$ ). Interestingly, this nomogram also calculates the predicted survival benefit from CRT according to the ypTNM stage. Expectedly, both the OHSU and Shapiro calculators had the highest level of agreement (ICC $=0.72$ ). The Sun and RPCCC risk calculators, by contrast, showed a lower performance (AUC $=0.53$ and 0.52 , respectively), even though these included a higher number of variables in their models.

It is challenging to explain the reasons underlying the non-optimal performances achieved by the calculators herein compared. It is possible that the variables selected in each calculator are not the most suitable and/or more are needed to provide accurate estimates of survival rate. Additionally, the diagnostic accuracy of clinical staging may have influenced the performance of those models that consider it as a prognostic factor. Furthermore, proposed nCRT agents and regimens differed among patients, as well as not every patient completed their neoadjuvant treatment. Thus, the low performance and agreement among calculators is possibly biased by these unaccounted differences. However, the development of clinical prediction tools has been applied to a myriad of other site-specific neoplasms, as in colorectal cancer, where calculators achieved good results in predicting 5-year OS, with RPCCC model being the one that achieving the best performance (AUC $=0.91$ ) (33).

Despite the difficulty for explanation of our results and uncertainty of how variables affect survival prediction, this study is important as it provides the first attempt at external validation for these nomograms. External validation is critical for these calculators as this is the avenue for improvement and expansion to a clinical tool that can be used across patient populations. The RPCCC achieved the 

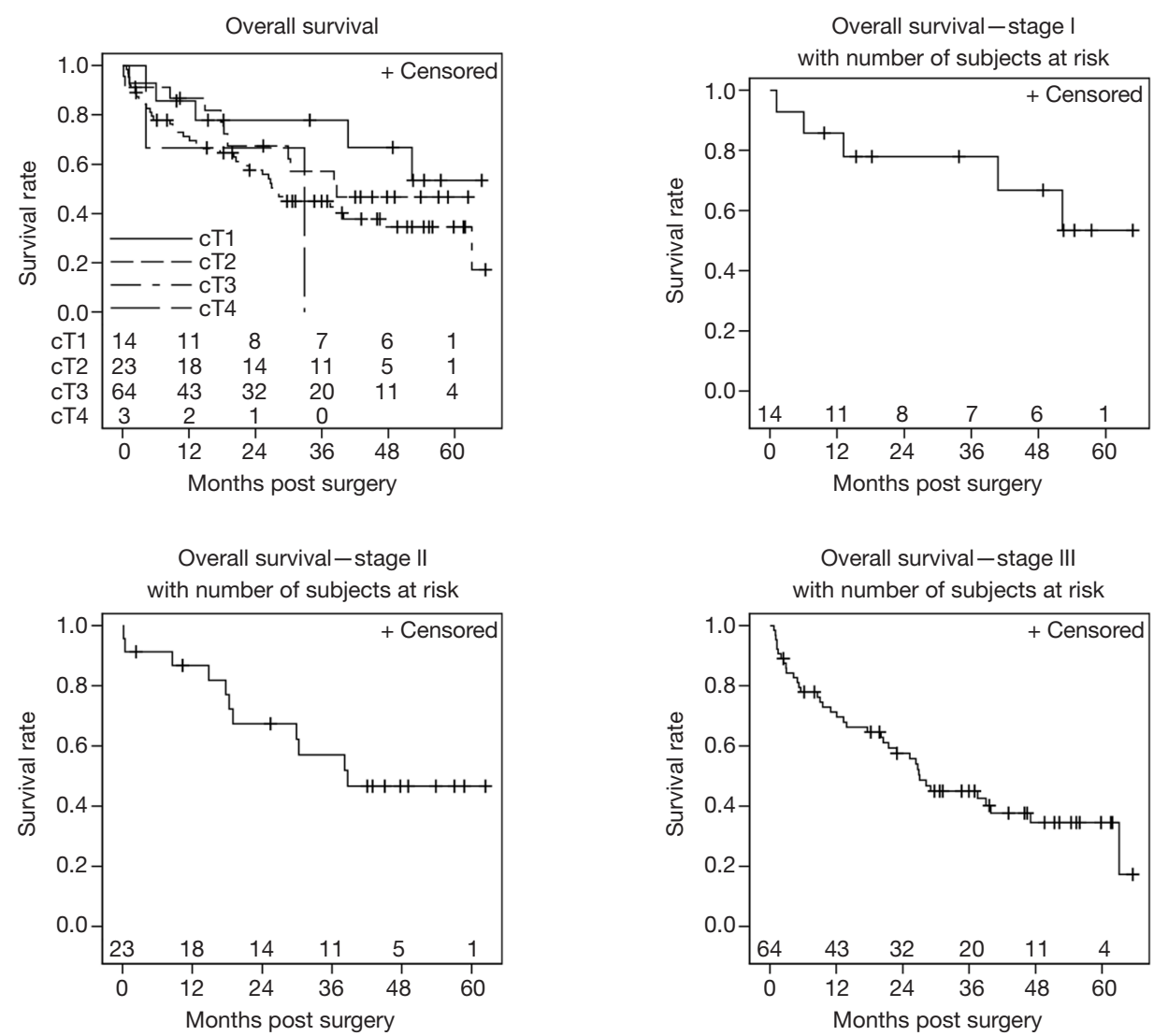

Figure 2 Kaplan-Meier curve showing 5-year OS for all-comers as well as by stage. cT, clinical T stage; OS, overall survival.

highest performance in a systematic review that evaluated nomograms with the Critical Appraisal and Data Extraction for Systematic Reviews of Prediction Modelling Studies, the gold standard for nomogram comparison (34). Our study used a different, yet valid, comparison analysis. Our study shows the need for improvement of a superior calculator (RPCCC) due to the poor performance in our external validation of different statistical comparison to other nomograms. Future steps to improve the RPCCC nomogram should incorporate additional data and variables along with artificial intelligence (AI). AI is an emerging concept that may aid in diagnosis, prediction, treatment decision making and response (35). In terms of predicting outcomes, AI may be superior to a traditional TMN staging. By incorporating AI into a prediction nomogram, increased precision and accuracy can be achieved regardless the evaluation metric.

There are a number of limitations to our study. As with any retrospective study, this comparison may introduce bias in patient selection. An additional limitation to this study is that there are not clearly defined methods for the clinical diagnosis for $\mathrm{T}$ and $\mathrm{N}$ status. Commonly, clinical diagnosis is reported based on imaging [positron emission tomography (PET)-computed tomography (CT), magnetic resonance imaging (MRI), endoscopy] and biopsy, providing histological diagnosis. However, the specific preoperative diagnosis was not reported in our database. There was also a limited number of patients $(n=104)$ in the available data which, subsequently, may alter the strength of the comparison. Nonetheless, our assessment of model performance was based on the AUC and calibration plots, which are unbiased and provide reasonable interval coverage even with just under 60 events. In this analysis, we did not compare all existing calculators that estimate survival rates for EC. For instance, the nomogram proposed by Xie et al. (25) for non-metastatic EC patients was not included because they selected patients who underwent only preoperative radiotherapy. Thus, it was not comparable to the population comprised in the calculators used. The prognostic models developed by Liu 


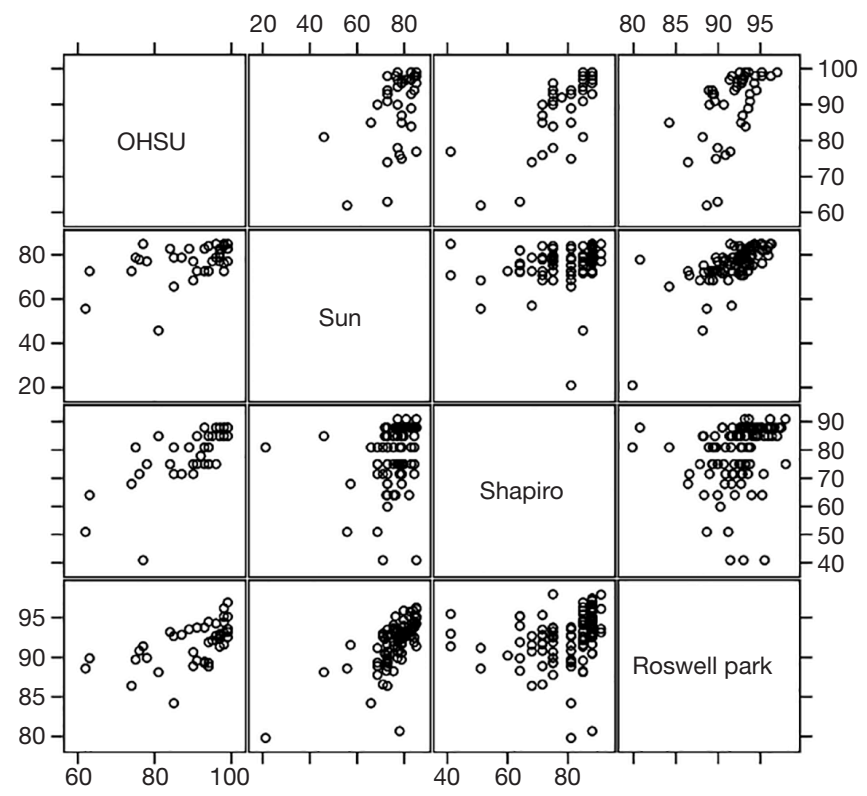

\begin{tabular}{|lcc|}
\hline Risk models & ICC & Spearman correlation coefficient \\
\hline Overall (all models) & 0.008 & - \\
\hline OHSU + Sun & 0.352 & $0.37(P=0.02)$ \\
\hline OHSU + Shapiro & 0.719 & $0.80(P<0.01)$ \\
\hline OHSU + Roswell Park & -0.055 & $0.61(P<0.01)$ \\
\hline Sun + Shapiro & 0.220 & $0.41(P<0.01)$ \\
\hline Sun + Roswell Park & 0.359 & $0.71(P<0.01)$ \\
\hline Shapiro + Roswell Park & 0.101 & $0.40(P<0.01)$ \\
\hline
\end{tabular}

Figure 3 Scatter plot matrix showing agreement between calculators. ICC, intra-class coefficient; OHSU, Oregon Health \& Science University.

et al. and Tang et al. $(21,24)$ included patients with M1 disease of EC. In the same way, the validated nomogram performed by Custodio et al. (36) was excluded because it focused on patients with unresectable locally advanced or metastatic adenocarcinoma of the distal esophagus, gastroesophageal junction, and stomach. Thus, these were all excluded from the comparison. Furthermore, predicting and comparing 1-year OS may provide limited applicability to clinical practice. Lastly, these equations and nomograms do not estimate OS instead of disease-free or disease-specific survival, which act as valid surrogates for OS (37) and provide more specific information about mortality rates directly related to cancer. However, the vast majority of recurrences in EA occur within 2 to 3 years of resection; therefore, in this study it would not be expected to differ significantly from the 1-year OS.

Despite these limitations, our analysis provides a valued juxtaposition among four EC survival nomogram models. We included patients from a multi-site Institution located in three different geographic regions in the United States, that provides important heterogeneity of the studied population, in addition to a robust statistical analysis. The Shapiro calculator estimated 1-year OS with superior performance, while the highest level of agreement was obtained by Shapiro and OHSU models, which share ypT and ypN stage as prognostic factors. The RPCCC model performed poorly in our external validation study despite superior performance in past studies. Subsequent steps will be taken to address our study's limitations and determine if higher performance can be achieved among these calculators. 

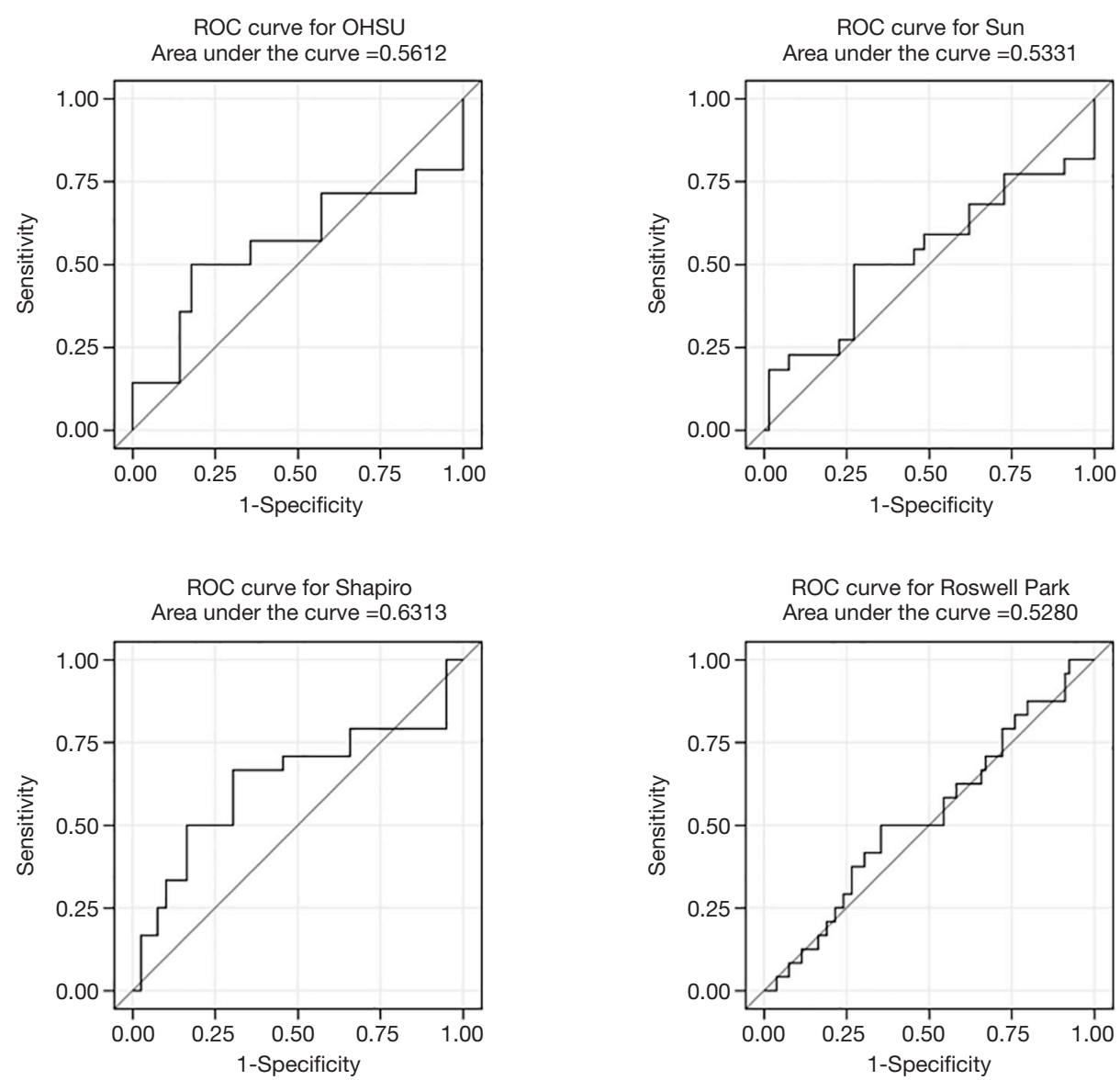

Figure 4 ROC curves showing calculators' performance. ROC, receiver operating characteristic.

Future plans include the addition of more patients prospectively, inclusion of $\mathrm{AI}$, and increasing variables to include genetic/biologic variables that will likely be related to response to neoadjuvant chemotherapy and survival outcomes. In conclusion, although these tools may be used as a part of the decision-making process for EC patients, their value and implementation in clinical practice requires additional refinement to optimize their clinical utility.

\section{Acknowledgments}

We acknowledge and thank the American College of Surgeons Committee on Cancer for providing access to the Participant User File from the National Cancer Data Base.

Funding: This work was supported by Roswell Park Cancer Institute and National Cancer Institute (NCI) grant P30CA016056; and the Mayo Clinic Robert D. and Patricia E. Kern Center for the Science of Health Care Delivery.

\section{Footnote}

Reporting Checklist: The authors have completed the STROBE reporting checklist. Available at https://dx.doi. org/10.21037/jgo-20-337

Data Sharing Statement: Available at https://dx.doi. org/10.21037/jgo-20-337

Conflicts of Interest: All authors have completed the ICMJE uniform disclosure form (available at https://dx.doi. org/10.21037/jgo-20-337). Dr. RL reports grants from Mayo Clinic Robert D. and Patricia E. Kern Center for the Science of Health Care Delivery, during the conduct of the study. Dr. TDV reports grants from Mayo Clinic Robert D. and Patricia E. Kern Center for the Science of Health Care Delivery, during the conduct of the study. Dr. KA reports grants from Roswell Park Cancer Institute and National Cancer Institute, during the conduct of the study; 
and employment at Roswell Park Comprehensive Cancer Center. Dr. ACS reports grants from Mayo Clinic Robert D. and Patricia E. Kern Center for the Science of Health Care Delivery, during the conduct of the study. Dr. DTC reports grants from Mayo Clinic Robert D. and Patricia E. Kern Center for the Science of Health Care Delivery, during the conduct of the study. Dr. MM reports grants from Roswell Park Cancer Institute and National Cancer Institute, during the conduct of the study; and employment at Roswell Park Comprehensive Cancer Center. Dr. EG reports grants from Roswell Park Cancer Institute and National Cancer Institute (NCI), from Mayo Clinic Robert D. and Patricia E. Kern Center for the Science of Health Care Delivery, during the conduct of the study. The other authors have no conflicts of interest to declare.

Disclaimer: The American College of Surgeons Committee on Cancer provided the Participant User File from the National Cancer Data Base, but has not reviewed or validated the results or conclusions of our study.

Ethical Statement: The authors are accountable for all aspects of the work in ensuring that questions related to the accuracy or integrity of any part of the work are appropriately investigated and resolved. The study was conducted in accordance with the Declaration of Helsinki (as revised in 2013). The study was deemed exempt by the institutional review board of Mayo Clinic Florida, and individual consent for this retrospective analysis was waived as this retrospective study was deemed minimal risk to patients.

Open Access Statement: This is an Open Access article distributed in accordance with the Creative Commons Attribution-NonCommercial-NoDerivs 4.0 International License (CC BY-NC-ND 4.0), which permits the noncommercial replication and distribution of the article with the strict proviso that no changes or edits are made and the original work is properly cited (including links to both the formal publication through the relevant DOI and the license). See: https://creativecommons.org/licenses/by-nc-nd/4.0/.

\section{References}

1. Siegel RL, Miller KD, Jemal A. Cancer statistics, 2015. CA Cancer J Clin 2015;65:5-29.

2. Siegel RL, Miller KD, Jemal A. Cancer statistics, 2018. CA Cancer J Clin 2018;68:7-30.

3. Long E, Beales IL. The role of obesity in oesophageal cancer development. Therap Adv Gastroenterol 2014;7:247-68.

4. Hoyo C, Cook MB, Kamangar F, et al. Body mass index in relation to oesophageal and oesophagogastric junction adenocarcinomas: a pooled analysis from the International BEACON Consortium. Int J Epidemiol 2012;41:1706-18.

5. Löfdahl HE, Lane A, Lu Y, et al. Increased population prevalence of reflux and obesity in the United Kingdom compared with Sweden: a potential explanation for the difference in incidence of esophageal adenocarcinoma. Eur J Gastroenterol Hepatol 2011;23:128-32.

6. Turati F, Tramacere I, La Vecchia C, et al. A meta-analysis of body mass index and esophageal and gastric cardia adenocarcinoma. Ann Oncol 2013;24:609-17.

7. Gibson MK, Dhaliwal AS, Clemons NJ, et al. Barrett's esophagus: cancer and molecular biology. Ann N Y Acad Sci 2013;1300:296-314.

8. van Hagen $\mathrm{P}$, Hulshof MC, van Lanschot JJ, et al. Preoperative chemoradiotherapy for esophageal or junctional cancer. N Engl J Med 2012;366:2074-84.

9. Walsh TN, Grennell M, Mansoor S, et al. Neoadjuvant treatment of advanced stage esophageal adenocarcinoma increases survival. Dis Esophagus 2002;15:121-4.

10. Shapiro J, van Lanschot JJB, Hulshof MCCM, et al. Neoadjuvant chemoradiotherapy plus surgery versus surgery alone for oesophageal or junctional cancer (CROSS): long-term results of a randomised controlled trial. Lancet Oncol 2015;16:1090-8.

11. Tepper J, Krasna MJ, Niedzwiecki D, et al. Phase III trial of trimodality therapy with cisplatin, fluorouracil, radiotherapy, and surgery compared with surgery alone for esophageal cancer: CALGB 9781. J Clin Oncol 2008;26:1086-92.

12. Mariette C, Piessen G, Briez N, et al. The number of metastatic lymph nodes and the ratio between metastatic and examined lymph nodes are independent prognostic factors in esophageal cancer regardless of neoadjuvant chemoradiation or lymphadenectomy extent. Ann Surg 2008;247:365-71.

13. Hofstetter W, Correa AM, Bekele N, et al. Proposed modification of nodal status in AJCC esophageal cancer staging system. Ann Thorac Surg 2007;84:365-73; discussion 374-5.

14. Rice TW, Blackstone EH, Rusch VW. 7th edition of the AJCC Cancer Staging Manual: esophagus and esophagogastric junction. Ann Surg Oncol 2010;17:1721-4.

15. Rice TW, Patil DT, Blackstone EH. 8th edition AJCC/UICC staging of cancers of the esophagus and 
esophagogastric junction: application to clinical practice. Ann Cardiothorac Surg 2017;6:119-30.

16. Davies AR, Gossage JA, Zylstra J, et al. Tumor stage after neoadjuvant chemotherapy determines survival after surgery for adenocarcinoma of the esophagus and esophagogastric junction. J Clin Oncol 2014;32:2983-90.

17. Vendrely V, Launay V, Najah H, et al. Prognostic factors in esophageal cancer treated with curative intent. Dig Liver Dis 2018;50:991-6.

18. Zarean E, Mahmoudi M, Azimi T, et al. Determining Overall Survival and Risk Factors in Esophageal Cancer Using Censored Quantile Regression Asian Pac J Cancer Prev 2018;19:3081-6.

19. Eil R, Diggs BS, Wang SJ, et al. Nomogram for predicting the benefit of neoadjuvant chemoradiotherapy for patients with esophageal cancer: a SEER-Medicare analysis. Cancer 2014;120:492-8.

20. Gabriel E, Attwood K, Shah R, et al. Novel Calculator to Estimate Overall Survival Benefit from Neoadjuvant Chemoradiation in Patients with Esophageal Adenocarcinoma. J Am Coll Surg 2017;224:884-894.e1.

21. Liu M, Wang C, Gao L, et al. A nomogram to predict long-time survival for patients with $\mathrm{M} 1$ diseases of esophageal cancer. J Cancer 2018;9:3986-90.

22. Shapiro J, van Klaveren D, Lagarde SM, et al. Prediction of survival in patients with oesophageal or junctional cancer receiving neoadjuvant chemoradiotherapy and surgery. Br J Surg 2016;103:1039-47.

23. Sun Y, Wang J, Li Y, et al. Nomograms to predict survival rates for esophageal cancer patients with malignant behaviors based on ICD-0-3. Future Oncol 2019;15:121-32.

24. Tang X, Zhou X, Li Y, et al. A Novel Nomogram and Risk Classification System Predicting the Cancer-Specific Survival of Patients with Initially Diagnosed Metastatic Esophageal Cancer: A SEER-Based Study. Ann Surg Oncol 2019;26:321-8.

25. Xie K, Liu S, Liu J. Nomogram predicts survival benefit for non- metastatic esophageal cancer patients who underwent preoperative radiotherapy. Cancer Manag Res 2018;10:3657-68.

26. Ychou M, Boige V, Pignon JP, et al. Perioperative chemotherapy compared with surgery alone for resectable gastroesophageal adenocarcinoma: an FNCLCC and FFCD multicenter phase III trial. J Clin Oncol 2011;29:1715-21.

27. Medical Research Council Oesophageal Cancer Working Group. Surgical resection with or without preoperative chemotherapy in oesophageal cancer: a randomised controlled trial. Lancet 2002;359:1727-33.

28. Cunningham D, Allum WH, Stenning SP, et al. Perioperative chemotherapy versus surgery alone for resectable gastroesophageal cancer. $\mathrm{N}$ Engl J Med 2006;355:11-20.

29. Walsh TN, Noonan N, Hollywood D, et al. A comparison of multimodal therapy and surgery for esophageal adenocarcinoma. N Engl J Med 1996;335:462-7.

30. Singla S, Gabriel E, Alnaji R, et al. Complete pathologic response is independent of the timing of esophagectomy following neoadjuvant chemoradiation for esophageal cancer. J Gastrointest Oncol 2018;9:73-9.

31. Gu Y, Swisher SG, Ajani JA, et al. The number of lymph nodes with metastasis predicts survival in patients with esophageal or esophagogastric junction adenocarcinoma who receive preoperative chemoradiation. Cancer 2006;106:1017-25.

32. Rizk NP, Venkatraman E, Bains MS, et al. American Joint Committee on Cancer staging system does not accurately predict survival in patients receiving multimodality therapy for esophageal adenocarcinoma. J Clin Oncol 2007;25:507-12.

33. Lemini R, Attwood K, Pecenka S, et al. Stage II-III colon cancer: a comparison of survival calculators. J Gastrointest Oncol 2018;9:1091-8.

34. Gupta V, Coburn N, Kidane B, et al. Survival prediction tools for esophageal and gastroesophageal junction cancer: A systematic review. J Thorac Cardiovasc Surg 2018;156:847-56.

35. Zhang YH, Guo LJ, Yuan XL, et al. Artificial intelligenceassisted esophageal cancer management: Now and future. World J Gastroenterol 2020;26:5256-71.

36. Custodio A, Carmona-Bayonas A, Jiménez-Fonseca P, et al. Nomogram-based prediction of survival in patients with advanced oesophagogastric adenocarcinoma receiving first-line chemotherapy: a multicenter prospective study in the era of trastuzumab. Br J Cancer 2017;116:1526-35.

37. Abrams J. Disease-free survival versus overall survival as a primary end point for adjuvant colon cancer studies: a commentary. J Clin Oncol 2005;23:8564-5.

Cite this article as: Lemini R, Díaz Vico T, Trumbull DA, Attwood K, Spaulding AC, Elli EF, Colibaseanu DT, Kukar M, Gabriel E. Prognostic models for stage I-III esophageal cancer: a comparison between existing calculators. J Gastrointest Oncol 2021;12(5):1963-1972. doi: 10.21037/jgo-20-337 